\title{
L'indépendance de la recherche peut-elle sacrifier la protection des sujets?
}

\author{
D. Sprumont
}

1 Aapro MS, Leyvraz S. L'indépendance de la recherche clinique survivra-t-elle? Revue médicale suisse 2005;1(20):1323-4. Sur le même ton dramatique: Malacrida R. Das Ende der freien Krebsforschung? Bioethica Forum 2003; (39):1-2.

Correspondance:

Pr Dr Dominique Sprumont Institut de droit de la santé

Université de Neuchâtel et Fribourg

Avenue du $1^{\text {er }}$ Mars 26

CH-2000 Neuchâtel

E-mail:

dominique.sprumont@unine.ch
Le $1^{\text {er }}$ janvier 2002 entrait en vigueur en Suisse la nouvelle loi sur les produits thérapeutiques. Le $1^{\mathrm{er}}$ mai 2004, c'était le tour de la nouvelle directive européenne 2001/20/CE sur les essais cliniques de médicament dans l'Union européenne. Cette «nouvelle» réglementation a levé toute ambiguité sur le fait que les études avec des produits thérapeutiques - quelle que soit leur source de financement - devaient se conformer aux mêmes règles de protection des sujets humains, mais aussi de qualité et de scientificité. D'un point de vue juridique, ces législations n'ont fait que clarifier la situation, sans modifier sur le fond la réglementation de la recherche biomédicale. En effet, il n'est ni moralement, ni juridiquement défendable que les personnes participant à un essai clinique jouissent d'une moindre protection sous prétexte que l'étude n'a pas de but lucratif.

Pourtant, d'aucuns vont aujourd'hui jusqu'à crier au loup en annonçant la fin de la recherche indépendante en Suisse et en Europe [1]. Par recherche indépendante, entendez ici celle qui n'est pas financée par l'industrie et qui n'est donc pas a priori entachée de conflits d'intérêts. Il est vrai qu'en pratique, les recherches sans but lucratif n'étaient souvent pas soumises de manière systématique aux mêmes contrôles que les études de l'industrie pharmaceutique. Dès le début des années nonante, les compagnies pharmaceutiques ont formellement intégré les règles des Bonnes pratiques des essais cliniques (Good Clinical Practice, GCP), avec pour maîtres mots le respect de procédures standard opérationnelles et la garantie de la traçabilité à chaque étape de l'étude. Une partie du milieu académique a été moins sensible à ce changement. Un embryon de formation en matière de GCP commence à se développer dans les facultés de médecine et les hôpitaux universitaires seulement depuis deux ou trois ans. Les recherches sans but lucratif se sont ainsi poursuivies selon des critères moins exigeants, principalement par omission des principaux intéressés, à savoir les investigateurs et les fonds de recherche. Ainsi, le Fonds national de la recherche scientifique a expressément refusé en 1993 d'assumer son rôle de sponsor lors de l'adoption du règlement de l'OICM sur les médicaments au stade d'essai clinique.
La «nouvelle» législation met un terme à cette dichotomie et garantit ainsi une protection adéquate aux sujets de recherche indépendamment de la finalité commerciale ou non des études. En pratique, les fonds de recherche sans but lucratif doivent aujourd'hui rattraper leur retard en assumant le coût d'une protection des sujets conforme à leurs droits fondamentaux et au respect de leur dignité. Le moment est mal choisi car les budgets de recherche sont soumis

\section{La couverture des risques: un défi} pour la recherche et l'innovation Depuis l'entrée en vigueur de la nouvelle loi sur les produits thérapeutiques, plusieurs cas ont révélé la difficulté pour les chercheurs travaillant en dehors de l'industrie pharmaceutique à faire face à leurs responsabilités et à obtenir une couverture d'assurance RC à un prix supportable. Une telle réalité se retrouve en Europe suite à l'introduction de la Directive 2000/21 sur les essais cliniques de médicaments. D'aucuns s'inquiètent des blocages que cela pourrait entraîner pour des recherches importantes du point de vue de la santé publique. Il paraît ainsi urgent de clarifier l'ensemble des enjeux liés à la couverture des risques dans le cadre d'essais cliniques et d'explorer des solutions pour le futur, aussi bien dans le cadre légal actuel qu'en prévision de la future loi fédérale sur la recherche impliquant des êtres humains.

Ces questions seront abordées par les principaux experts juridiques suisses dans ce domaine, ainsi que les spécialistes des questions d'assurance RC et des essais cliniques. La conférence est destinée en particulier aux chercheurs, aux fonds publics de recherche, à l'industrie pharmaceutique, aux hôpitaux, aux autorités cantonales et fédérales de santé publique, ainsi qu'aux commissions d'éthique de la recherche.

Pour plus d'information: www.unine.ch/ ids ou forum gesundheitsrecht, Madame Brigitte Wittwer, Case postale 769, Mühlebachstrasse 32, 8024 Zurich, tél. 04426620 86, e-mail: wittwer@bdp.ch. 
plus que jamais à des restrictions. Il est alors tentant d'économiser sur la protection des sujets.

$\mathrm{S}$. Radczuweit et ses collègues mettent ainsi en lumière les problèmes fréquents rencontrés en matière de contrat d'assurance RC. Cette question pose toujours plus de problèmes. L'affaire la plus grave a été celle de la SIAK dont il est avéré que pendant plusieurs années elle a mené des études en oncologie sans couverture d'assurance RC. En l'espèce, il ne s'agissait pas vraiment d'une question budgétaire - la SIAK payait des primes d'assurance (sic) - mais de connaissance de la loi. En effet, a priori, la SIAK n'avait tout simplement pas conscience de ses obligations légales.

La situation est aujourd'hui particulièrement préoccupante. Une solution doit être trouvée au découragement qui frappe certains chercheurs. La recherche indépendante est essentielle et elle peut et doit se poursuivre dans le respect des lois. Des efforts importants de formation et d'organisation doivent ainsi être consentis. L'Etat doit d'ailleurs s'impliquer davantage pour jouer pleinement son rôle en soutenant mieux les structures de contrôle des essais cliniques et en évitant ainsi de grever les fonds de recherche. Des aménagements doivent peut-être aussi être apportés à la loi. L'Union européenne vient ainsi d'adopter une nouvelle directive 2005/28/CE qui reconnaît la spécificité des essais cliniques non commerciaux. En Suisse, le projet de loi sur la recherche biomédicale, qui devrait être mis en consultation cette année encore, ne manquera pas de tenir compte des problèmes actuels.

D'ici là, il convient de renforcer le dialogue entre les investigateurs, les commissions d'éthique de la recherche et Swissmedic, mais aussi entre l'industrie et les fonds publics de recherche. Les 29 et 30 septembre se tiendra ainsi à Zurich, une conférence sur les questions de responsabilité civile et d'assurance RC dans la recherche biomédicale. Patronnée par l'Académie suisse des sciences médicales, l'Office fédéral de la santé publique, Swissmedic et la SwissRe, cette manifestation vise à chercher des solutions à la crise actuelle, mais aussi à développer de nouvelles pistes de réflexion pour le futur.

\section{Risikodeckung: Eine Herausforderung} für Forschung und Innovation

Seit das neue Heilmittelgesetz in Kraft getreten ist, haben verschiedentlich Fälle von Forschenden, die nicht in die Pharmaindustrie eingebettet sind, die Schwierigkeiten dieser Personen aufgezeigt, eine erschwingliche Versicherungsdeckung im Bereich der Privathaftpflicht zu erlangen. Im europäischen Raum ist man seit der Einführung der Richtlinien 2000/21 über die klinischen Medikamentenversuche mit der gleichen Realität konfrontiert. Doch scheint niemand besorgt zu sein um die Schwierigkeiten und Hindernisse, welche dadurch in wichtigen Forschungsprojekten entstehen können. Es besteht dringlicher Klärungsbedarf bezüglich verschiedener Fragen, die mit der Risikodeckung im Rahmen der klinischen Versuche zusammenhängen. Auch scheint es notwendig, Lösungsansätze zu erarbeiten, dies sowohl im Bereich der geltenden gesetzlichen Rahmenbedingungen wie im Hinblick auf das zukünftige Bundesgesetz über die Forschung am Menschen.

Diese Fragen werden von den wichtigsten Schweizer Rechtsexperten des Gesundheitsrechts und der Spezialisten der Privathaftpflichtversicherung und der klinischen Versuche erörtert werden. Die Konferenz richtet sich insbesondere an Forschende, die öffentlichen Forschungsfonds, die pharmazeutische Industrie, die Spitäler, die kantonalen und eidgenössischen Behörden des Gesundheitswesens wie auch die Ethikkommissionen für klinische Forschung.

Für mehr Information: www.unine.ch/ids oder forum gesundheitsrecht, Brigitte Wittwer, Postfach 769, Mühlebachstrasse 32, 8024 Zürich, Tel. 04426620 86, E-Mail: wittwer@ bdp.ch. 